\title{
On the existence, structure and stability of static and stationary solutions of the Einstein-Vlasov system
}

\author{
Håkan Andréasson \\ Mathematical Sciences \\ Chalmers University of Technology \\ University of Gothenburg \\ S-41296 Göteborg, Sweden
}

\begin{abstract}
The present status on the existence, structure and stability of static and stationary solutions of the Einstein-Vlasov system is reviewed. Under the assumptions that a spherically symmetric static object has isotropic pressure and non-increasing energy density outwards, Buchdahl showed 1959 the bound $M / R<4 / 9$, where $M$ is the ADM mass and $R$ the outer radius. Most static solutions of the Einstein-Vlasov system do not satisfy these assumptions. The bound $\mathrm{M} / \mathrm{R}<4 / 9$ nevertheless holds and it is sharp. An analogous bound in the charged case is also given. The important question of stability of spherically symmetric static solutions is presently open but numerical results are available and these are reviewed. A natural question is to go beyond spherical symmetry and consider axially symmetric solutions, and a recent result on the existence of axially symmetric stationary solutions is also discussed.
\end{abstract}

Keywords: Einstein-Vlasov system, Buchdahl inequalities, axially symmetric spacetimes PACS: $04.20-\mathrm{q}, 04.40 \mathrm{Dg}$

\section{STATIONARY ASYMPTOTICALLY-FLAT SOLUTIONS}

Equilibrium states in galactic dynamics can be described as static or stationary solutions of the Einstein-Vlasov system, or of the Vlasov-Poisson system in the Newtonian case. Here we consider the relativistic case and we refer to the review paper [1] for the Newtonian case. First, the spherically-symmetric case for which the structure is well understood is discussed. The question of the stability of the spherically-symmetric static solutions of the Einstein-Vlasov system is basically open, which is in sharp contrast to the situation for the Vlasov-Poisson system. A numerical study on stability is reviewed. At the end the results $[2,31]$ on axisymmetric static solutions are presented. The equations become much more complicated by going beyond spherical symmetry and only solutions close to spherically symmetric ones have been analyzed. This review is to a large extent an extract from the the section on static and stationary solutions in the review article [3].

\section{Existence of spherically-symmetric static solutions}

For a general introduction to relativistic kinetic theory and the Einstein-Vlasov system I refer to [3] and the notation below follows the one given in [3].

Let us assume that spacetime is static and spherically symmetric. Let the metric have the form

$$
d s^{2}=-e^{2 \mu(r)} d t^{2}+e^{2 \lambda(r)} d r^{2}+r^{2}\left(d \theta^{2}+\sin ^{2} \theta d \varphi^{2}\right),
$$

where $r \geq 0, \theta \in[0, \pi], \varphi \in[0,2 \pi]$. As before, asymptotic flatness is expressed by the boundary conditions

$$
\lim _{r \rightarrow \infty} \lambda(r)=\lim _{r \rightarrow \infty} \mu(r)=0,
$$

and a regular center requires

$$
\lambda(0)=0 .
$$

The time-independent Einstein-Vlasov system then reads, cf. [3],

$$
e^{\mu-\lambda} \frac{v}{\sqrt{1+|v|^{2}}} \cdot \nabla_{x} f-\sqrt{1+|v|^{2}} e^{\mu-\lambda} \mu_{r} \frac{x}{r} \cdot \nabla_{v} f=0,
$$




$$
\begin{aligned}
& e^{-2 \lambda}\left(2 r \lambda_{r}-1\right)+1=8 \pi r^{2} \rho, \\
& e^{-2 \lambda}\left(2 r \mu_{r}+1\right)-1=8 \pi r^{2} p .
\end{aligned}
$$

The matter quantities are defined as:

$$
\begin{aligned}
& \rho(x)=\int_{R^{3}} \sqrt{1+|v|^{2}} f(x, v) d v \\
& p(x)=\int_{R^{3}}\left(\frac{x \cdot v}{r}\right)^{2} f(x, v) \frac{d v}{\sqrt{1+|v|^{2}}} .
\end{aligned}
$$

The quantities

$$
E:=e^{\mu(r)} \sqrt{1+|v|^{2}}, \quad L=|x|^{2}|v|^{2}-(x \cdot v)^{2}=|x \times v|^{2},
$$

are conserved along characteristics. $E$ is the particle energy and $L$ is the angular momentum squared. If we let

$$
f(x, v)=\Phi(E, L),
$$

for some function $\Phi$, the Vlasov equation is automatically satisfied.

A common assumption is to restrict the form of $\Phi$ to

$$
\Phi(E, L)=\phi(E)\left(L-L_{0}\right)_{+}^{l},
$$

where $l>-1 / 2, L_{0} \geq 0$ and $x_{+}=\max \{x, 0\}$. If we furthermore assume that

$$
\phi(E)=\left(E-E_{0}\right)_{+}^{k}, k>-1,
$$

for some positive constant $E_{0}$, then we obtain the polytropic ansatz. The case of isotropic pressure is obtained by letting $l=0$ so that $\Phi$ only depends on $E$.

In passing, we mention that for the Vlasov-Poisson system it has been shown [4] that every static sphericallysymmetric solution must have the form $f=\Phi(E, L)$. This is referred to as Jeans' theorem. It was an open question for some time whether or not this was also true for the Einstein-Vlasov system. This was settled in 1999 by Schaeffer [5], who found solutions that do not have this particular form globally on phase space, and consequently, Jeans' theorem is not valid in the relativistic case. However, almost all results on static solutions are based on this ansatz.

By inserting the ansatz $f(x, v)=\Phi(E, L)$ in the matter quantities $\rho$ and $p$, a non-linear system for $\lambda$ and $\mu$ is obtained, in which

$$
\begin{aligned}
& e^{-2 \lambda}\left(2 r \lambda_{r}-1\right)+1=8 \pi r^{2} G_{\Phi}(r, \mu), \\
& e^{-2 \lambda}\left(2 r \mu_{r}+1\right)-1=8 \pi r^{2} H_{\Phi}(r, \mu),
\end{aligned}
$$

where

$$
\begin{aligned}
& G_{\Phi}(r, \mu)=\frac{2 \pi}{r^{2}} \int_{1}^{\infty} \int_{0}^{r^{2}\left(\varepsilon^{2}-1\right)} \Phi\left(e^{\mu(r)} \varepsilon, L\right) \frac{\varepsilon^{2}}{\sqrt{\varepsilon^{2}-1-L / r^{2}}} d L d \varepsilon \\
& H_{\Phi}(r, \mu)=\frac{2 \pi}{r^{2}} \int_{1}^{\infty} \int_{0}^{r^{2}\left(\varepsilon^{2}-1\right)} \Phi\left(e^{\mu(r)} \varepsilon, L\right) \sqrt{\varepsilon^{2}-1-L / r^{2}} d L d \varepsilon .
\end{aligned}
$$

Existence of solutions to this system was first proven in the case of isotropic pressure in [6], and extended to anisotropic pressure in [7]. The main difficulty is to show that the solutions have finite ADM mass and compact support. The argument in these works to obtain a solution of compact support is to perturb a steady state of the Vlasov-Poisson system, which is known to have compact support. Two different types of solutions are constructed, those with a regular centre [6,7], and those with a Schwarzschild singularity in the centre [7].

In [8], Rein and Rendall go beyond the polytropic ansatz and obtain steady states with compact support and finite mass under the assumption that $\Phi$ satisfies

$$
\Phi(E, L)=c\left(E-E_{0}\right)_{+}^{k} L^{l}+O\left(\left(E_{0}-E\right)_{+}^{\delta+k}\right) L^{l} \text { as } E \rightarrow E_{0},
$$

where $k>-1, l>-1 / 2, k+l+1 / 2>0, k<l+3 / 2$. This result is obtained in a more direct way and is not based on the perturbation argument used in [6,7]. Their method is inspired by a work on stellar models by Makino [9], in which he considers steady states of the Euler-Einstein system. An alternative method to obtain steady states with finite radius and finite mass, which is based on a dynamical system analysis, is given in [10]. 


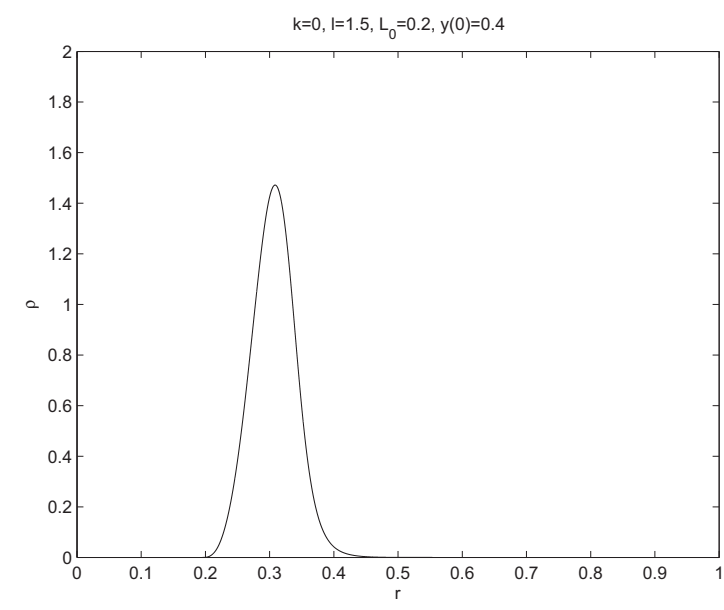

FIGURE 1.

\section{The structure of spherically-symmetric steady states}

All solutions described so far have the property that the support of $\rho$ contains a ball about the centre. In [11] Rein showed that steady states also exist whose support is a finite, spherically-symmetric shell with a vacuum region in the center. In [12] it was shown that there are shell solutions, which have an arbitrarily thin thickness. A systematic study of the structure of spherically-symmetric static solutions was carried out mainly by numerical means in [13] and we now present the conclusions of this investigation.

By prescribing the value $\mu(0)$, the equations can be solved, but the resulting solution will in general not satisfy the boundary condition $\mu(\infty)=0$, but it will have some finite limit. It is then possible to shift both the cut-off energy $E_{0}$ and the solution by this limit to obtain a solution, which satisfies $\mu(\infty)=0$. A convenient way to handle the problem that $E_{0}$ and $\mu(0)$ cannot both be treated as free parameters is to use the ansatz

$$
f(x, v)=\left(1-E / E_{0}\right)_{+}^{k}\left(L-L_{0}\right)_{+}^{l}, k \geq 0, l>-1 / 2, k<3 l+7 / 2,
$$

as in [13]. This gives an equation for $\mu$, which can be rewritten in terms of the function

$$
y(r)=\frac{e^{\mu(r)}}{E_{0}} .
$$

In this way the cut-off energy disappears as a free parameter of the problem and we thus have the four free parameters $k, l, L_{0}$ and $y(0)$. The structure of the static solutions obtained in [13] is as follows:

If $L_{0}=0$ the energy density can be strictly positive or vanish at $r=0$ (depending on $l$ ) but it is always strictly positive sufficiently close to $r=0$. Hence, the support of the matter is an interval $\left[0, R_{1}\right]$ with $R_{1}>0$, and we call such states ball configurations. If $L_{0}>0$ the support is in an interval $\left[R_{0}, R_{1}\right], R_{0}>0$, and we call such steady states for shells.

The value $y(0)$ determines how compact or relativistic the steady state is, and the smaller values the more relativistic. For large values, recall $y(0) \leq 1 / E_{0}$, a pure shell or a pure ball configuration is obtained, cf. Figure 1 for a pure shell. Note that we depict the behavior of $\rho$ but we remark that the pressure terms behave similarly but the amplitudes of $p$ and $p_{T}$ can be very different, i.e., the steady states can be highly anisotropic.

For moderate values of $y(0)$ the solutions have a distinct inner peak and a tail-like outer peak, and by making $y(0)$ smaller more peaks appear, cf. Figure 2 for the case of ball configurations.

In the case of shells there is a similar structure but in this case the peaks can either be separated by vacuum regions or by thin atmospheric regions as in the case of ball configurations. An example with multi-peaks, where some of the peaks are separated by vacuum regions, is given in Figure 3.

A different feature of the structure of static solutions is the issue of spirals. For a fixed ansatz of the density function $f$, there is a one-parameter family of static solutions, which are parameterized by $y(0)$. A natural question to ask is 

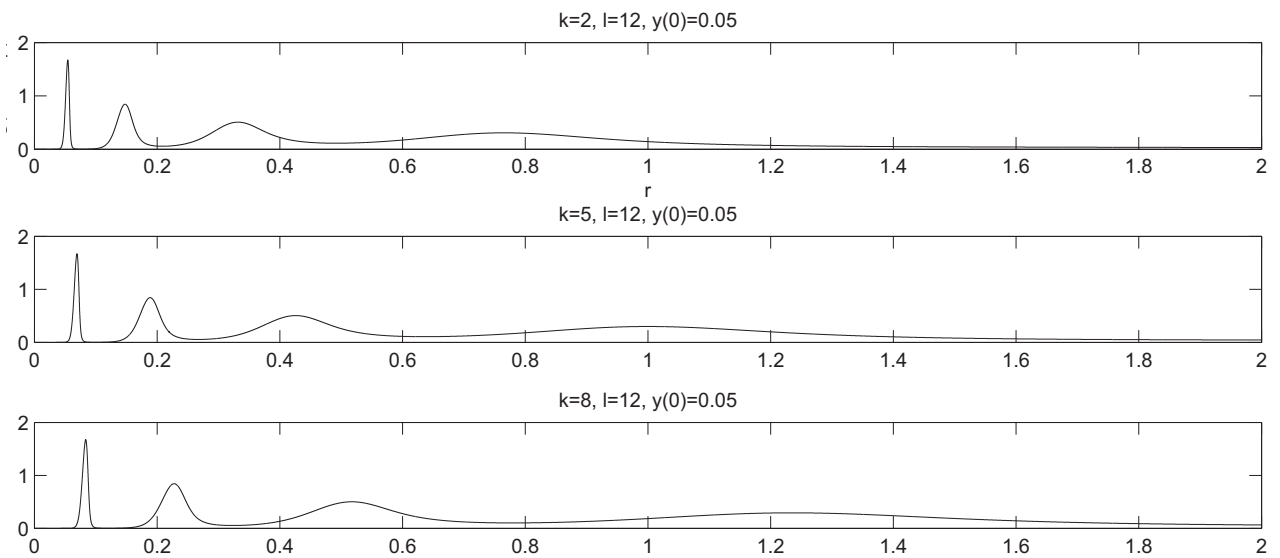

FIGURE 2.

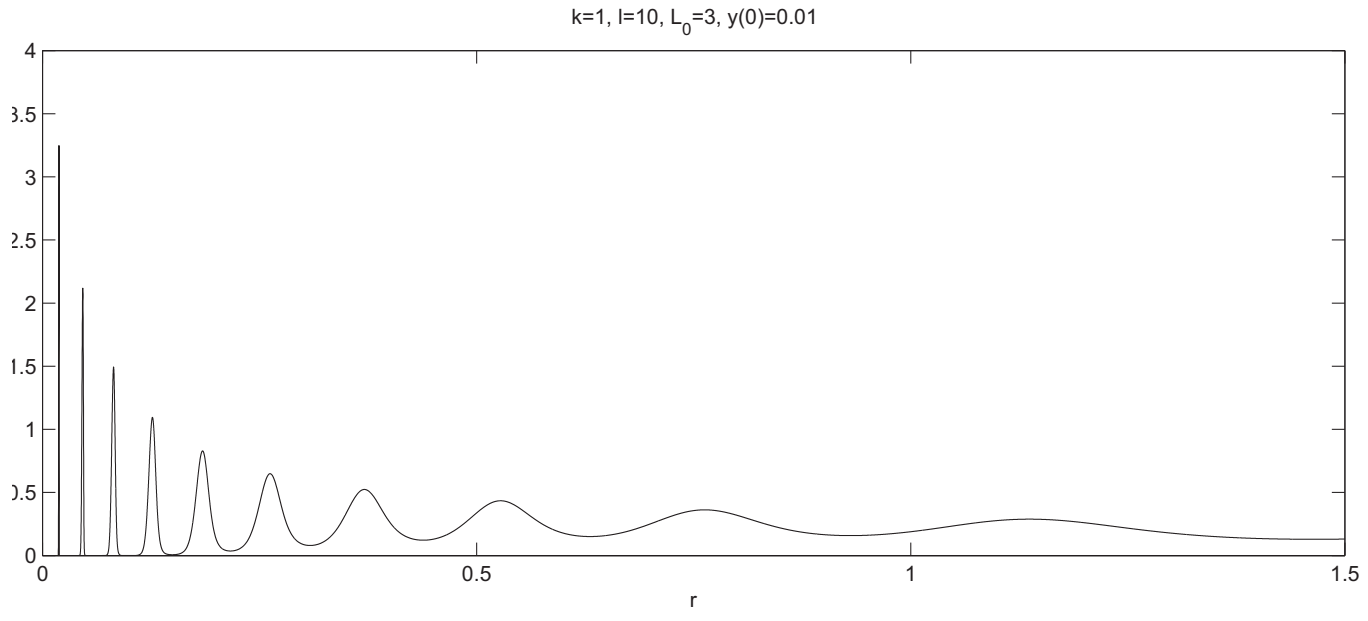

FIGURE 3.

how the ADM mass $M$ and the radius of the support $R$ change along such a family. By plotting for each $y(0)$ the resulting values for $R$ and $M$ a curve is obtained, which reflects how radius and mass are related along such a oneparameter family of steady states. This curve has a spiral form, cf. Figure 4. It is shown in [13] that in the isotropic case, where $l=L_{0}=0$ the radius-mass curves always have a spiral form. It should be pointed out that there is a clear distinction between solutions of the Einstein- Vlasov system and solutions of the Vlasov-Poisson system; in the former case the $M-R$ curve seems always to form a spiral whereas this is not so in the latter case. One reason why this issue is important is that in astrophysics conclusions about stability are often drawn from the "Poincaré turning point principle" based on a spiral diagram.

\section{Buchdahl-type inequalities}

Another aspect of the structure of steady states investigated numerically in [13] concerns the Buchdahl inequality. If a steady state has support in $\left[R_{0}, R_{1}\right]$, then the ADM mass $M$ of the configuration is $M=m\left(R_{1}\right)$, where $m(r)$ is the quasi local mass, cf. [3]. 


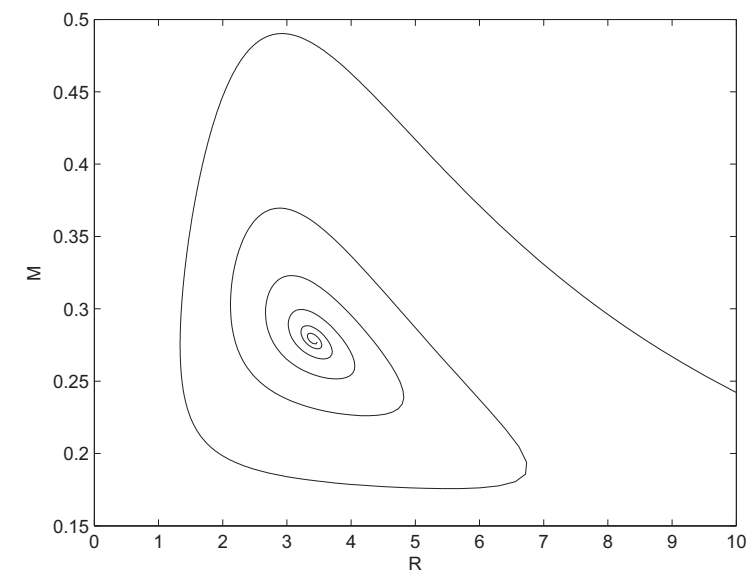

FIGURE 4.

Schwarzschild asked already in 1916 the question: How large can $2 M / R$ possibly be? He gave the answer [14] $2 M / R \leq 8 / 9$ in the special case of the Schwarzschild interior solution, which has constant energy density and isotropic pressure. In 1959 Buchdahl [15] extended his result to isotropic solutions for which the energy density is non-increasing outwards and he showed that also in this case

$$
\frac{2 M}{R} \leq \frac{8}{9}
$$

This is sometimes called the Buchdahl inequality. Let us remark that the Buchdahl inequality can obviously be written as $M / R \leq 4 / 9$, but since it is the quantity $2 M / R$, which appears in the Schwarzschild metric, it is common to keep the form of Equation (8). A bound on $2 M / R$ has an immediate observational consequence since it limits the possible gravitational red shift of a spherically-symmetric static object.

The assumptions made by Buchdahl are very restrictive. In particular, the overwhelming number of the steady states of the Einstein-Vlasov system have neither an isotropic pressure nor a non-increasing energy density, but nevertheless $2 M / R$ is always found to be less than $8 / 9$ in the numerical study [13]. Also for other matter models the assumptions are not satisfying. As pointed out by Guven and Ó Murchadha [16], neither of the Buchdahl assumptions hold in a simple soap bubble and they do not approximate any known topologically stable field configuration. In addition, there are several astrophysical models of stars, which are anisotropic, e.g. Lemâitre [17] proposed a model of an anisotropic star already in 1933. Hence, it is an important question to investigate bounds on $2 M / R$ under less restrictive assumptions.

In [18] it is shown that for any static solution of the spherically-symmetric Einstein equation, not necessarily of the Einstein-Vlasov system, for which $p \geq 0$, and

$$
p+2 p_{T} \leq \Omega \rho
$$

the following inequality holds

$$
\frac{2 m(r)}{r} \leq \frac{(1+2 \Omega)^{2}-1}{(1+2 \Omega)^{2}}
$$

Moreover, the inequality is sharp and sharpness is obtained uniquely by an infinitely thin shell solution. Note in particular that for Vlasov matter $\Omega=1$ and that the right-hand side then equals $8 / 9$ as in the Buchdahl inequality. An alternative proof was given in [19] and their method applies to a larger class of conditions on $\rho, p$ and $p_{T}$ than the one given in Equation (9). On the other hand, the result in [19] is weaker than the result in [18] in the sense that the latter method implies that the steady state that saturates the inequality is unique; it is an infinitely thin shell. The studies $[18,19]$ are of general character and in particular it is not shown that solutions exist to the coupled Einstein-matter system, which can saturate the inequality. For instance, it is natural to ask if there are solutions of the Einstein-Vlasov system, which have $2 \mathrm{~m} / \mathrm{r}$ arbitrarily close to 8/9. This question is given an affirmative answer in [12], 
where in particular it is shown that arbitrarily thin shells exist, which are regular solutions of the spherically-symmetric Einstein-Vlasov system. Using the strategy in [20] it follows that

$$
\sup _{r} \frac{2 m(r)}{r} \rightarrow \frac{8}{9}
$$

in the limit when the shells become infinitely thin.

The question of finding an upper bound on $2 M / R$ can be extended to charged objects and to the case with a positive cosmological constant. The spacetime outside a spherically-symmetric charged object is given by the ReissnerNordström metric

$$
d s^{2}=-\left(1-\frac{2 M}{r}+\frac{Q}{r^{2}}\right) d t^{2}+\left(1-\frac{2 M}{r}+\frac{Q}{r^{2}}\right)^{-1} d r^{2}+r^{2}\left(d \theta^{2}+\sin ^{2} \theta d \phi^{2}\right),
$$

where $Q$ is the total charge of the object. The quantity $1-\frac{2 M}{r}+\frac{Q}{r^{2}}$ is zero when $r_{ \pm}=M \pm \sqrt{M^{2}-Q^{2}}$, and $r_{ \pm}$is called the inner and outer horizon respectively of a Reissner-Nordström black hole. A Buchdahl type inequality gives a lower bound of the area radius of a static object and this radius is thus often called the critical stability radius. It is shown in [21] that a spherically-symmetric static solution of the Einstein-Maxwell system for which $p \geq 0, p+2 p_{T} \leq \rho$, and $Q<M$ satisfy

$$
\sqrt{M} \leq \frac{\sqrt{R}}{3}+\sqrt{\frac{R}{9}+\frac{Q^{2}}{3 R}} .
$$

Note, in particular, that the inequality holds for solutions of the Einstein-Vlasov-Maxwell system, since the conditions above are always satisfied in this case. This inequality (11) implies that the stability radius is outside the outer horizon of a Reissner-Nordström black hole. In [22] the relevance of an inequality of this kind on aspects in black-hole physics is discussed. In contrast to the case without charge, the saturating solution is not unique. An infinitely thin shell solution does saturate the inequality (11), but numerical evidence is given in [23] that there is also another type of solution, which saturates the inequality for which the inner and outer horizon coincide.

The study in [24] is concerned with the non-charged situation when a positive cosmological constant $\Lambda$ is included. The following inequality is derived

$$
\frac{M}{R} \leq \frac{2}{9}-\frac{\Lambda R^{2}}{3}+\frac{2}{9} \sqrt{1+3 \Lambda R^{2}}
$$

for solutions for which $p \geq 0, p+2 p_{T} \leq \rho$, and $0 \leq \Lambda R^{2} \leq 1$. In this situation, the question of sharpness is essentially open. An infinitely thin shell solution does not generally saturate the inequality but does so in the two degenerate situations $\Lambda R^{2}=0$ and $\Lambda R^{2}=1$. In the latter case there is a constant density solution, and the exterior spacetime is the Nariai solution, which saturates the inequality and the saturating solution is thus non-unique. In this case, the cosmological horizon and the black hole horizon coincide, which is in analogy with the charged situation described above where the inner and outer horizons coincide when uniqueness is likely lost.

\section{Stability}

An important problem is the question of the stability of spherically-symmetric steady states. At present, there are few theoretical results on the stability of the steady states of the Einstein-Vlasov system. Wolansky [25] has applied the energy-Casimir method and obtained some insights, but the theory is much less developed than in the Vlasov-Poisson case and the nonlinear stability problem is open. The situation is very different for the Vlasov-Poisson system, and we refer to [1] for a review on the results in this case.

However, there are numerical studies [26, 27, 28] on the stability of spherically-symmetric steady states for the Einstein-Vlasov system. The latter two studies concern isotropic steady states, whereas the first, in addition, treats anisotropic steady states. Here we present the conclusions of [26], emphasizing that these agree with the conclusions in $[28,27]$ for isotropic states.

To allow for trapped surfaces, maximal-areal coordinates are used, i.e., the metric is written in the following form in [26]

$$
d s^{2}=-\left(\alpha^{2}-a^{2} \beta^{2}\right) d t^{2}+2 a^{2} \beta d t d r+a^{2} d r^{2}+r^{2}\left(d \theta^{2}+\sin ^{2} \theta d \phi^{2}\right) .
$$

Here the metric coefficients $\alpha, \beta$, and $a$ depend on $t \in \mathbf{R}$ and $r \geq 0, \alpha$ and $a$ are positive, and the polar angles $\theta \in[0, \pi]$ and $\phi \in[0,2 \pi]$ parameterize the unit sphere. Thus, the radial coordinate $r$ is the area radius. A maximal 


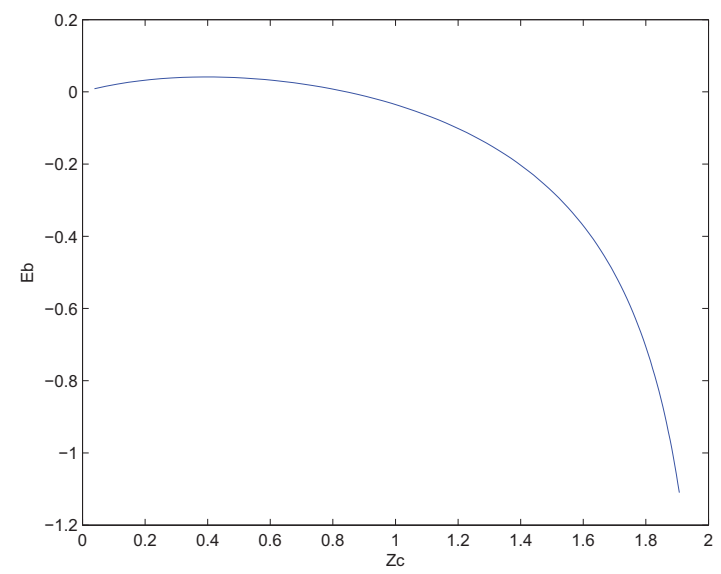

FIGURE 5.

gauge condition is then imposed, which means that each hypersurface of constant $t$ has vanishing mean curvature. The boundary conditions, which guarantee asymptotic flatness and a regular center, are given by

$$
a(t, 0)=a(t, \infty)=\alpha(t, \infty)=1
$$

Steady states are numerically constructed, and these are then perturbed in order to investigate the stability. More precisely, to construct the steady states the polytropic ansatz is used, cf. Section,

$$
f(r, w, L)=\Phi(E, L)=\left(E_{0}-E\right)_{+}^{k}\left(L-L_{0}\right)_{+}^{l} .
$$

By specifying values on $E_{0}, L_{0}$ and $\alpha(0)$ steady states are numerically constructed. The distribution function $f_{s}$ of the steady state is then multiplied by an amplitude $A$, so that a new, perturbed distribution function is obtained. This is then used as initial datum in the evolution code. We remark that also other types of perturbations are analyzed in [26].

For $k$ and $l$ fixed each steady state is characterized by its central red shift $Z_{c}$ and its fractional binding energy $E_{b}$, which are defined by

$$
Z_{c}=\frac{1}{\alpha(0)}-1, E_{b}=\frac{e_{b}}{M_{0}}, \text { where } e_{b}=M_{0}-M
$$

Here

$$
M_{0}=4 \pi^{2} \int_{0}^{\infty} \int_{-\infty}^{\infty} \int_{0}^{\infty} a(t, r) f(t, r, w, L) d L d w d r
$$

is the total number of particles, which, since all particles have rest mass one, equals the rest mass of the system. $M$ is the ADM mass given by

$$
M=\int_{0}^{\infty}\left(4 \pi \rho(t, r)+\frac{3}{2} \kappa^{2}(t, r)\right) r^{2} d r
$$

where $\kappa=\beta / r \alpha$. Both $M_{0}$ and $M$ are conserved quantities. The central redshift is the redshift of a photon emitted from the center and received at infinity, and the binding energy $e_{b}$ is the difference of the rest mass and the ADM mass. In Figure 5 and Figure 6 the relation between the fractional binding energy and the central redshift is given for two different cases.

The relevance of these concepts for the stability properties of steady states was first discussed by Zel'dovich and Podurets [29], who argued that it should be possible to diagnose the stability from binding energy considerations. Zel'dovich and Novikov [30] then conjectured that the binding energy maximum along a steady state sequence signals the onset of instability.

The picture that arises from the simulations in [26] is summarized in Table 1. Varying the parameters $k, l$ and $L_{0}$ give rise to essentially the same tables, cf. [26]. 


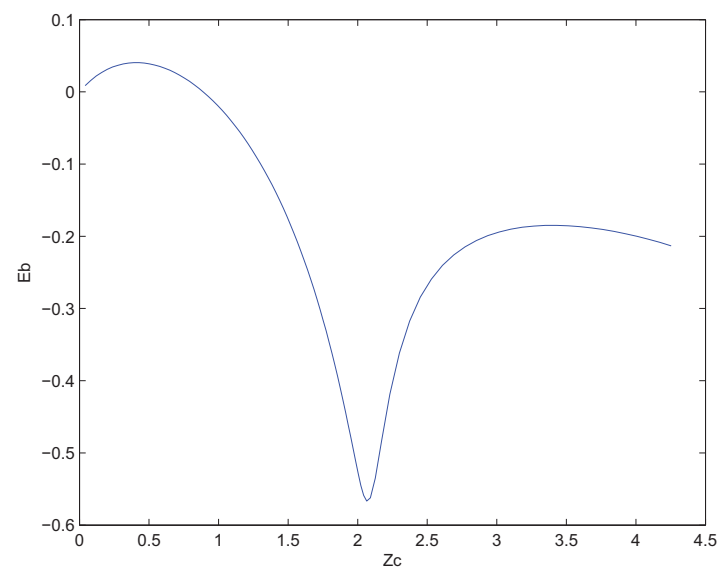

FIGURE 6.

TABLE 1. $k=0$ and $l=1 / 2$.

$\begin{array}{rrrc}Z_{c} & E_{b} & A<1 & A>1 \\ 0.21 & 0.032 & \text { stable } & \text { stable } \\ 0.34 & 0.040 & \text { stable } & \text { stable } \\ 0.39 & 0.040 & \text { stable } & \text { stable } \\ 0.42 & 0.041 & \text { stable } & \text { unstable } \\ 0.46 & 0.040 & \text { stable } & \text { unstable } \\ 0.56 & 0.036 & \text { stable } & \text { unstable } \\ 0.65 & 0.029 & \text { stable } & \text { unstable } \\ 0.82 & 0.008 & \text { stable } & \text { unstable } \\ 0.95 & -0.015 & \text { unstable } & \text { unstable } \\ 1.20 & -0.078 & \text { unstable } & \text { unstable }\end{array}$

If we first consider perturbations with $A>1$, it is found that steady states with small values on $Z_{c}$ (less than approximately 0.40 in this case) are stable, i.e., the perturbed solutions stay in a neighbourhood of the static solution. A careful investigation of the perturbed solutions indicates that they oscillate in a periodic way. For larger values of $Z_{c}$ the evolution leads to the formation of trapped surfaces and collapse to black holes. Hence, for perturbations with $A>1$ the value of $Z_{c}$ alone seems to determine the stability features of the steady states. Plotting $E_{b}$ versus $Z_{c}$ with higher resolution, cf. [26], gives support to the conjecture by Novikov and Zel'dovich mentioned above that the maximum of $E_{b}$ along a sequence of steady states signals the onset of instability.

The situation is quite different for perturbations with $A<1$. The crucial quantity in this case is the fractional binding energy $E_{b}$. Consider a steady state with $E_{b}>0$ and a perturbation with $A<1$ but close to 1 so that the fractional binding energy remains positive. The perturbed solution then drifts outwards, turns back and reimplodes, and comes close to its initial state, and then continues to expand and reimplode and thus oscillates, cf. Figure 7. Whether or not these oscillations are periodic in time is an open and very interesting question.

In [28] it is stated (without proof) that if $E_{b}>0$ the solution must ultimately reimplode and the simulations in [26] support that it is true. For negative values of $E_{b}$, the solutions with $A<1$ disperse to infinity.

A simple analytic argument is given in [26], which relates the question, whether a solution disperses or not. It is shown that if a shell solution has an expanding vacuum region of radius $R(t)$ at the center with $R(t) \rightarrow \infty$ for $t \rightarrow \infty$, i.e., the solution disperses in a strong sense, then necessarily $M_{0} \leq M$, i.e., $E_{b} \leq 0$. 

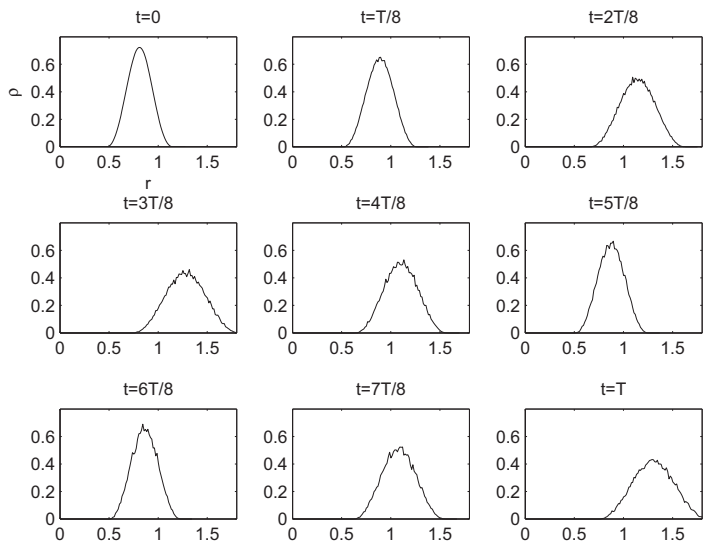

FIGURE 7.

\section{Existence of axisymmetric static solutions}

As we have seen above, a broad variety of static solutions of the Einstein-Vlasov system has been established, all of which share the restriction that they are spherically symmetric. The investigations $[2,31]$ remove this restriction and prove the existence of static and stationary solutions of the Einstein-Vlasov system, which are axially symmetric but not spherically symmetric. The solutions constructed in [31] have non-vanishing total angular momentum whereas the solutions in [2] have vanishing angular momentum. From the applications point of view axial symmetry is more "realistic" than spherical symmetry, and from the mathematics point of view the complexity of the Einstein field equations increases drastically if one gives up spherical symmetry. Before discussing these results, let us mention that similar results have been obtained for two other matter models. In the case of a perfect fluid, Heilig showed the existence of axisymmetric stationary solutions in [32]. These solutions have non-zero angular momentum since static solutions are necessarily spherically symmetric. In this respect the situation for elastic matter is more similar to Vlasov matter. The existence of static axisymmetric solutions of elastic matter, which are not spherically symmetric, was proven in [33]. Stationary solutions with rotation were then established in [34].

Let us now briefly discuss the method of proof in [2], which relies on an application of the implicit function theorem. The method of proof in [31] is analogous with an additional difficulty which we briefly discuss at the end.

The set-up of the problem follows the work of Bardeen [35], where the metric is written in the form

$$
d s^{2}=-c^{2} e^{2 v / c^{2}} d t^{2}+e^{2 \mu} d \rho^{2}+e^{2 \mu} d z^{2}+\rho^{2} B^{2} e^{-2 v / c^{2}} d \varphi^{2}
$$

for functions $v, B, \mu$ depending on $\rho$ and $z$, where $t \in \mathbf{R}, \rho \in\left[0, \infty\left[, z \in \mathbf{R}\right.\right.$ and $\varphi \in[0,2 \pi]$. The Killing vector fields $\partial_{t}$ and $\partial_{\phi}$ correspond to the stationarity and axial symmetry of the spacetime. Solutions are obtained by perturbing off spherically symmetric steady states of the Vlasov-Poisson system via the implicit function theorem and the reason for writing $v / c^{2}$ in the metric, instead of $v$, is that $v$ converges to the Newtonian potential $U_{N}$ of the steady state in the limit $c \rightarrow \infty$. Asymptotic flatness is expressed by the boundary conditions

$$
\lim _{|(\rho, z)| \rightarrow \infty} v(\rho, z)=\lim _{|(\rho, z)| \rightarrow \infty} \mu(\rho, z)=0, \lim _{|(\rho, z)| \rightarrow \infty} B(\rho, z)=1 .
$$

In addition the solutions are required to be locally flat at the axis of symmetry, which implies the condition

$$
v(0, z) / c^{2}+\mu(0, z)=\ln B(0, z), z \in \mathbf{R} .
$$

Let us now recall from Section the strategy to construct static solutions by using an ansatz of the form

$$
f(x, v)=\Phi(E, L)
$$


where $E$ and $L$ are conserved quantities along characteristics. Due to the symmetries of the metric (15) the following quantities are constant along geodesics:

$$
\begin{aligned}
E & :=-g\left(\partial / \partial t, p^{a}\right)=c^{2} e^{2 v / c^{2}} p^{0} \\
& =c^{2} e^{v / c^{2}} \sqrt{1+c^{-2}\left(e^{2 \mu}\left(p^{1}\right)^{2}+e^{2 \mu}\left(p^{2}\right)^{2}+\rho^{2} B^{2} e^{\left.-2 v / c^{2}\left(p^{3}\right)^{2}\right)}\right.} \\
L & :=g\left(\partial / \partial \varphi, p^{a}\right)=\rho^{2} B^{2} e^{-2 v / c^{2}} p^{3} .
\end{aligned}
$$

Here $p^{a}$ are the canonical momenta. $E$ can be thought of as a local or particle energy and $L$ is the angular momentum of a particle with respect to the axis of symmetry. For a sufficiently regular $\Phi$ the ansatz function $f$ satisfies the Vlasov equation and upon insertion of this ansatz into the definition of the energy momentum tensor the latter becomes a functional $T_{\alpha \beta}=T_{\alpha \beta}(\nu, B, \mu)$ of the unknown metric functions $v, B, \mu$. It then remains to solve the Einstein equations with this energy momentum tensor as right-hand side. The Newtonian limit of the Einstein-Vlasov system is the Vlasov-Poisson system and the strategy in [2] is to perturb off spherically symmetric steady states of the VlasovPoisson system via the implicit function theorem to obtain axisymmetric solutions. Indeed, the main result of [2] specifies conditions on the ansatz function $\Phi$ such that a two parameter $(\gamma$ and $\lambda)$ family of axially-symmetric solutions of the Einstein-Vlasov system passes through the corresponding spherically symmetric, Newtonian steady state, whose ansatz function we denote by $\phi$. The parameter $\gamma=1 / c^{2}$ turns on general relativity and the parameter $\lambda$ turns on the dependence on $L$. Since $L$ is not invariant under arbitrary rotations about the origin the solution is not spherically symmetric if $f$ depends on $L$. It should also be mentioned that although $\gamma$ is a priori small, which means that $c$ is large, the scaling symmetry of the Einstein-Vlasov system can be used to obtain solutions corresponding to the physically correct value of $c$. The most striking condition on the ansatz function $\Phi$, or rather on the ansatz function $\phi$ of the corresponding Vlasov-Poisson system, needed to carry out the proof is that it must satisfy

$$
6+4 \pi r^{2} a_{N}(r)>0, \quad r \in[0, \infty[,
$$

where

$$
a_{N}(r):=\int_{\mathbf{R}^{3}} \phi^{\prime}\left(\frac{1}{2}|v|^{2}+U_{N}(r)\right) d v .
$$

An important argument in the proof is indeed to justify that there are steady states of the Vlasov-Poisson system satisfying this condition.

In [31] this result is extended to stationary solutions with rotation. The metric takes the form

$$
d s^{2}=-c^{2} e^{2 v / c^{2}} d t^{2}+e^{2 \mu} d \rho^{2}+e^{2 \mu} d z^{2}+\rho^{2} B^{2} e^{-2 v / c^{2}}(d \varphi-\omega d t)^{2},
$$

and the new difficulty in this case is to treat the equation for the additional metric function $\omega$ which is required to admit non-vanishing total angular momentum. The linearized $\omega$-equation takes the form

$$
\partial_{\rho \rho} \omega+\partial_{z z} \omega+\frac{3}{\rho} \partial_{\rho} \omega=q
$$

for a suitable class of right-hand sides $q$. The difficulty with this fairly innocent looking elliptic equation is that the coefficient $3 / \rho$ blows up on the axis of symmetry, where the full solution must remain smooth. The following formula gives a solution of the $\omega$ equation:

$$
\omega(\rho, z)=\int_{0}^{\infty} \int_{R} q(\tilde{\rho}, \tilde{z}) \mathscr{K}(\rho, z, \tilde{\rho}, \tilde{z}) d \tilde{z} d \tilde{\rho},
$$

where

$$
\mathscr{K}(\rho, z, \tilde{\rho}, \tilde{z}):=-\frac{1}{2 \pi}\left(\frac{\tilde{\rho}}{\rho}\right)^{3 / 2} Q_{1 / 2}\left(\frac{\rho^{2}+\tilde{\rho}^{2}+(z-\tilde{z})^{2}}{2 \rho \tilde{\rho}}\right),
$$

with $Q_{1 / 2}$ denoting the half-integer Legendre function of the second kind. The analysis which is needed to obtain the required regularity and decay properties is very demanding but possible to achieve. However, the linearized $\omega$ equation is nothing but the Poisson equation on $R^{5}$ where both $\omega$ and $q$ are axially symmetric, i.e., they depend on $\rho=\left|\left(x_{1}, x_{2}, x_{3}, x_{4}\right)\right|$ and $z=x_{5}$. The solution formula is now standard and the required regularity and decay estimates follow by well-known theory. An analogous observation applies to the linearized equation for $B$ which can be turned into the Poisson equation on $R^{4}$. This simplifies the proof and improves the result also for vanishing angular momentum. 


\section{REFERENCES}

1. G. Rein, "Collisionless kinetic equations from astrophysics - the Vlasov-Poisson system," in Handbook of Differential Equations: Evolutionary Equations, vol. 3, edited by C. Dafermos, and E. Feireisl, Elsevier/North-Holland, Amsterdam, 2006, pp. 383-476.

2. H. Andréasson, M. Kunze, and G. Rein, Commun. Math. Phys. 308, $23-47$ (2011).

3. H. Andréasson, Living Rev. Relativity 14 (2011).

4. J. Batt, W. Faltenbacher, and E. Horst, Arch. Ration. Mech. Anal. 93, 159-183 (1986).

5. J. Schaeffer, Commun. Math. Phys. 204, 313-327 (1999).

6. G. Rein, and A. Rendall, Ann. Inst. Henri Poincare A 59, 383-397 (1993).

7. G. Rein, Math. Proc. Camb. Phil. Soc. 115, 559-570 (1994).

8. G. Rein, and A. Rendall, Math. Proc. Camb. Phil. Soc. 128, 363-380 (2000).

9. T. Makino, J. Math. Kyoto Univ. 38, 55-69 (1998).

10. M. Fjällborg, M. Heinzle, and C. Uggla, Math. Proc. Camb. Phil. Soc. 143, 731-752 (2007).

11. G. Rein, Indiana Univ. Math. J. 48, 335-346 (1999).

12. H. Andréasson, Commun. Math. Phys. 274, 409-425 (2007).

13. H. Andréasson, and G. Rein, Class. Quantum Grav. 24, 1809-1832 (2007).

14. K. Schwarzschild, Sitzungsber. K. Preuss. Akad. Wiss., Phys.-Math. Kl. 1916, 424-434 (1916).

15. H. Buchdahl, Phys. Rev. 116, 1027-1034 (1959).

16. J. Guven, and N. ÓMurchadha, Phys. Rev. D 60 (1999).

17. G. Lemaitre, Ann. Soc. Sci. Bruxelles, Ser. A 53, 51 (1993).

18. H. Andréasson, J. Differ. Equations 245, 2243-2266 (2008).

19. P. Karageorgis, and J. Stalker, Class. Quantum Grav. 25 (2008).

20. H. Andréasson, Commun. Math. Phys. 274, 399-408 (2007).

21. H. Andréasson, Commun. Math. Phys. 288, 715-730 (2009).

22. A. Giuliani, and T. Rothman, Gen. Relativ. Gravit. 40, 1427-1447 (2008).

23. H. Andréasson, M. Eklund, and G. Rein, Class. Quantum Grav. 26 (2009).

24. H. Andréasson, and C. Böhmer, Class. Quantum Grav. 26, 1-11 (2009).

25. G. Wolansky, Arch. Ration. Mech. Anal. 156, 205-230 (2001).

26. H. Andréasson, and G. Rein, Class. Quantum Grav. 23, 3659-3677 (2006).

27. J. Ipser, Astrophys. J. 158, 17-43 (1969).

28. S. Shapiro, and S. Teukolsky, Astrophys. J. 298, 58-79 (1985).

29. Y. Zel'dovich, and M. Podurets, Sov. Astron. 9, 742-749 (1965), translated from Astronomicheskii Zhurnal 42, 963-973 (1965).

30. Y. Zel'dovich, and I. Novikov, Relativistic Astrophysics, vol. 1, University of Chicago Press, Chicago, 1971.

31. H. Andréasson, M. Kunze, and G. Rein, To appear in Commun. Math. Phys. (????).

32. U. Heilig, CommuMath, n.. Phys. 166, 457-493 (1995).

33. L. Andersson, R. Beig, and B. Schmidt, Commun. Pure Appl. Math. 61, 988-1023 (2008).

34. L. Andersson, R. Beig, and B. Schmidt, Commun. Pure Appl. Math. 63, 559-589 (2009).

35. J. Bardeen, "Rapidly rotating stars, disks, and black holes," in Black Holes, edited by C. DeWitt, and B. DeWitt, Gordon and Breach, New York, U.S.A., 1973, pp. 241-289. 